\title{
Historia de los anestesiólogos en el Centro Médico ABC
}

\section{Anesthesiologist history at the ABC Medical Center}

\author{
Paulina Seguí Vizcaíno*
}

Citar como: Seguí VP. Historia de los anestesiólogos en el Centro Médico ABC. An Med ABC. 2021; 66 (3): 229-232. https://dx.doi. org/10.35366/101673

\begin{abstract}
RESUMEN
El artículo resume cerca de 70 años de historia de los anestesiólogos dentro del Centro Médico ABC. Algunos de los logros alcanzados durante estos años son: organizar una asociación para la compra de máquinas de anestesia y monitores, desarrollar un programa de residencia médica, colaborar en la organización de varios departamentos dentro de la institución, ocupar puestos en el área administrativa y médica, entre otros. Todo esto se logra gracias al trabajo en equipo de los anestesiólogos, en colaboración con otras especialidades y administrativos del hospital. Actualmente se viven tiempos con retos nuevos que requieren mayor trabajo en equipo multidisciplinario y aprendizaje virtual, siempre con el objetivo de brindarle al paciente una práctica de la anestesiología segura, sustentable y eficiente.
\end{abstract}

Palabras clave: Historia anestesiólogos, sociedad de anestesiólogos, residencia anestesia, Centro de Capacitación de la American Heart Association.

La historia del Centro Médico ABC empieza en 1866 con el Hospital Americano y uniéndose al Sanatorio Cowdray en 1941 formó lo que se conocía como el Hospital Inglés. ${ }^{1}$ Los médicos de esa época anestesiaban, operaban y vigilaban al paciente, eran mé-

\footnotetext{
* Anestesióloga y egresada del Centro Médico ABC. Presidente actual de práctica médica grupal (PMG) Anestesiólogos ABC. México.
}

Recibido: 03/06/2021. Aceptado: 26/08/2021.

Correspondencia: Dra. Paulina Seguí Vizcaíno

E-mail: pauseg2@yahoo.com.mx

\begin{abstract}
This article summarizes the history of anesthesia at the American British Cowdray Hospital. The first group of anesthesiologists organized well and consequently funded the purchase of anesthetic machines and monitors systems. With time, the growing number of anesthesiologists formed a residence program which began in 1989. Subsequently, the department of anesthesia began to contribute with other areas inside the institution, academically and administratively. Today as a group, new challenges have arisen with technology, networking and teaching, all of which are currently focused on patient safety while practicing sustainable and efficiently the anesthesia.
\end{abstract}

Keywords: Anesthesiologist history, anesthesiologist society, anesthesia residence, American Heart Association Mexico Center.

dicos generales que realizaban todas las funciones junto con las enfermeras. En el edificio original en la colonia Anzures existía una sola sala de operaciones, en la foto encontrada cabe destacar que no hay máquina de anestesia ni monitores, simplemente una camilla, un lavabo y una mesa de mayo, la lámpara era de pie y todo de azulejos. ${ }^{2}$ Cuando el hospital se muda a Observatorio en 1964 se construyen cuatro salas de operaciones en la planta baja y aquí inicia la historia contemporánea del departamento de anestesiología.

Dos anestesiólogos figuran en la lista de socios fundadores activos de la Primera Sociedad Médica 
del ABC Hospital (1955): ${ }^{3}$ Dr. Pablo Hawk del Rosal y el Dr. Fernando Zorrilla y junto con los doctores Enrique Hülsz, Alfonso Cota, Alejandro de Ávila, Rafael Álvarez González, Eduardo Shain, Petricciolli, Carlos Peón y varios más se constituyó la Sociedad de Anestesiólogos del Hospital ABC en 1975, a la cual tenían que pertenecer para ejercer dentro del hospital. Dicha sociedad se formó para poder comprar máquinas de anestesia y equipo, ya que el hospital no contaba con dichos aparatos y ellos, en búsqueda de contar con lo más moderno y seguro para los pacientes, decidieron comprarlas. A inicios de los años 80 cuando los doctores Pastor Luna, Carlos Hurtado, Rubén Valdespín, Hernández Fabela, Leopoldo Torres, Gerardo Álvarez Reséndiz, Raúl Salazar, Roger Luna, José Chapa, Adalberto Toro, Marco Antonio Chávez, entre otros, llegan al hospital, tienen que aportar el equivalente a una máquina de anestesia para poder ejercer dentro de la institución y los siguientes miembros sólo una cuota de recuperación. Este dinero era utilizado para dar mantenimiento a las máquinas y comenzar a comprar el equipo de monitoreo necesario para empezar a vigilar a los pacientes con algo más que un baumanómetro manual y un estetoscopio. Cabe mencionar que no existían los ventiladores y cada anestesiólogo llevaba su laringoscopio, tubos, Guedel y hasta Yankauer, todo era lavado y reutilizado, no se desechaba nada. Una caja de pescador era la fiel acompañante de todo anestesiólogo donde además de todos estos insumos, había medicamentos nuevos y en jeringas, lo que hoy es inconcebible para nuestra práctica hace tan sólo 60 años, era lo más moderno y seguro.

El primer jefe de servicio fue el Dr. Enrique Hülsz, después el Dr. Calvillo y el Dr. De Alva, mientras que ginecobstetricia siempre se mantuvo separada con una coordinación al inicio con el Dr. Arévalo, el Dr. Zorrilla y desde los años 80 con el Dr. Valdespín, que se encargan, entre otras cosas, de coordinar las guardias de 24 horas que existen para los procedimientos de urgencias. Y desde 1985 se crean roles de guardias de urgencias para que el hospital cuente con un anestesiólogo las 24 horas en cualquier servicio que se requiera uno.

En los años 70 los médicos que ingresaron a la institución habían realizado la subespecialidad en el extranjero como ejemplo la Dra. Estela Melman en anestesia pediátrica (primera mujer en ser aceptada como anestesióloga en 1968 y tercera en toda la Asociación Médica), el Dr. Enrique Hülsz y el Dr. Álvarez González en anestesia cardiovascular. Mientras que en los años 80 empezaron a llegar los anestesiólogos intensivistas que complementaron de manera importante la práctica y enseñanza de la especialidad como el Dr. Hilario Genovés y el Dr. Jaime Ortega.

En la década de 1980 el hospital es remodelado y se construyen siete quirófanos en primer piso y uno que se comparte en conjunto con ginecología donde había cuatro salas para cirugías y partos, siendo remodelados 10 años después debido a la alta demanda quirúrgica, aumentando a 10 salas, y es en este momento que la sociedad de anestesiólogos dona las máquinas de anestesia al hospital y empieza a hacerse cargo directamente de la compra y mantenimiento de las mismas así como de la compra de todos los monitores y cualquier otro equipo necesario para que nosotros los anestesiólogos podamos ejercer la práctica de nuestra especialidad como la conocemos el día de hoy. Durante esos años los anestesiólogos cambiaron la caja de pescador por unos carritos donde se guardaban además mascarillas laríngeas, fibroscopios, tubos especiales para cirugías de láser, circuitos de anestesia y se continuaban guardando medicamentos para no tener desecho de los mismos en jeringas membretadas manualmente con un plumón.

Un dato importante dentro de estas remodelaciones son los espacios de descanso y vestidores, donde las anestesiólogas hemos intervenido, especialmente la Dra. Ma. Teresa Esquinca en los años 90 logró que tuviéramos un espacio para las mujeres médicos separado de enfermería y proveedores, asimismo el Dr. Gerardo Bermúdez encontró espacios en ambos campus para que todos los médicos tengamos un gimnasio moderno, funcional con alimentos saludables.

En el año 2000 se comienza la construcción del Campus Santa Fe, se inaugura con cuatro salas de operaciones, incrementando a siete y contando con 15 salas para 2020; mientras que el Hospital de Ginecología y Obstetricia cuenta con cinco salas de operaciones, haciendo un total de 31 salas de operaciones en sus dos campus, con máquinas que se renuevan constantemente, que junto con un equipo de ingenieros biomédicos de 24 horas, proveen apoyo en cualquier falla del equipo. Más aún contamos con monitores no invasivos e invasivos de la más alta tecnología, material desechable para cada paciente, medicamentos individuales, y todo regido bajo los más altos estándares de calidad y en apego a las normas mexicanas e internacionales.

La evolución de la práctica de la anestesia es continua, se dejaron de dar anestesias y sedaciones en consultorios y por médicos ajenos a la especialidad como era en los años 80 . Y esta seguridad para el paciente también fue por insistencia de los jefes de ser- 
vicio y anestesiólogos en general, colaborando con las diferentes especialidades médicas.

En 1988 el Dr. Pastor Luna lleva personalmente los documentos a la UNAM para lograr consolidar la residencia de anestesiología dentro del hospital, siendo los primeros residentes en 1989: Gerardo Bermúdez, Ma. Teresa Esquinca y Fernando Rivadeneira, quienes realizaban guardias $\mathrm{A}-\mathrm{B}$, sin vacaciones durante tres años (en vez de sólo dos años como en el resto de las sedes y con ocho meses en terapia intensiva). Y a pesar de la diferencia de guardias, vacaciones y años, médicos intensivistas recién egresados «bajaron» los siguientes años a hacer la residencia (la unidad de terapia intensiva estaba en el tercer piso y los quirófanos en el primero): Horacio Olivares, Antonio Covarrubias, Cesar Zambada y más tarde muchos más. Los primeros egresados están por cumplir 30 años, y de ser una sede poco conocida hoy es una de las más buscadas por su alto nivel académico y excelente trato laboral.

En la Figura 1 se muestra el número de egresados activos dentro y fuera de la institución así como las defunciones. El programa de residencia ha contado con cuatro jefes a lo largo de estos años: Pastor Luna, Hilario Genovés, Carlos Hurtado, Marco Antonio Chávez y todos los miembros del personal que deseen contribuir en la formación académica de los residentes. Desde sus inicios, los maestros del curso han fomentado complementar los estudios y así han estudiado al terminar: cuidados críticos, cardiotorácica, neurología, perinatología, dolor, pediatría, tanatología y cuidados paliativos, siendo cerca de $50 \%$ con doble especialidad o diplomado.

Las salas de operaciones y de obstetricia no son los únicos espacios que ocupamos los anestesiólogos, el Dr. Elías Horta fue jefe de fisiología pulmonar e inhaloterapia de 1988 a 2004 y ocupó la vicepresidencia médica de 2004 a 2021. El Dr. C Hurtado fue director médico de 2009 a 2011. El Dr. Horacio Olivares fue tesorero de la Asociación Médica en 2002-2003. Hoy en día el Dr. Gerardo Bermúdez es el presidente de la Asociación Médica 20021-2023.

Un área también transformada por los anestesiólogos, actualmente a cargo del Dr. Jorge Romero, es el Centro de Capacitación de la American Heart Association del Centro Médico ABC (CMABC), su historia será publicada en breve y aquí sólo mencionaré que de ser un curso sólo para residentes en los años 90 teniendo que ser avalados por el Hospital Español, se consolidó en 2005 como centro reconocido por la American Heart, ocupando siempre los primeros seis espacios en Latinoamérica. Anualmente certifi-

ca y recertifica a toda la institución, tanto en BLS, PALS y ACLS cada dos años. Esto implica un enorme esfuerzo de instructores con una gran vocación de academia, de los que $60 \%$ son anestesiólogos que trabajan en equipo con intensivistas, internistas, urgenciólogos, pediatras y cardiólogos.

Como parte de la evolución de la anestesia es importante mencionar al Dr. Rodrigo Rubio que se capacita en el extranjero y colabora para la creación del Centro de Simulación del CMABC en 2012, donde residentes y personal de todas las especialidades continuamente se capacitan, impartiéndose alrededor de 60 diferentes simulaciones por año.

A su vez el Dr. Marco Antonio Chávez durante cuatro años hace consciente a la administración de la institución de que se requiere una Práctica Médica Grupal (PMG) como ya ocurría con otras especialidades de la institución y así el Dr. Alejandro Díaz es el primer presidente en 2017 de la PMG y con ello la Sociedad de Anestesiólogos, después de casi 60 años de organizar a los anestesiólogos, da por concluida su vigencia. Actualmente la PMG tiene alrededor de 170 colaboradores y es una de las mejor organizadas dentro de todas las prácticas que existen, gracias una vez más a la relación cordial que existe entre administrativos, jefatura y miembros de la práctica, los cuales son egresados del CMABC y de las instituciones públicas existentes en el país, siendo la mayoría de la CDMX.

Así vemos como a lo largo de los años (Figura 2) hemos ido ocupando diferentes espacios dentro de la institución, promoviendo la seguridad del paciente

115 residentes egresados del $A B C$

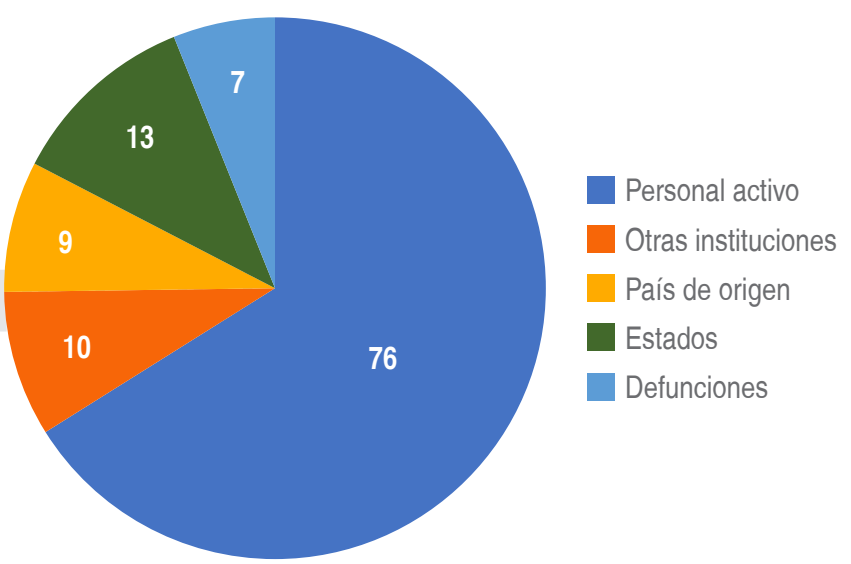

Figura 1: Número de residentes egresados de 1992 a 2021, egresados miembros del personal activos, egresados que regresaron a sus estados o países de origen, egresados en otras instituciones y egresados difuntos. 


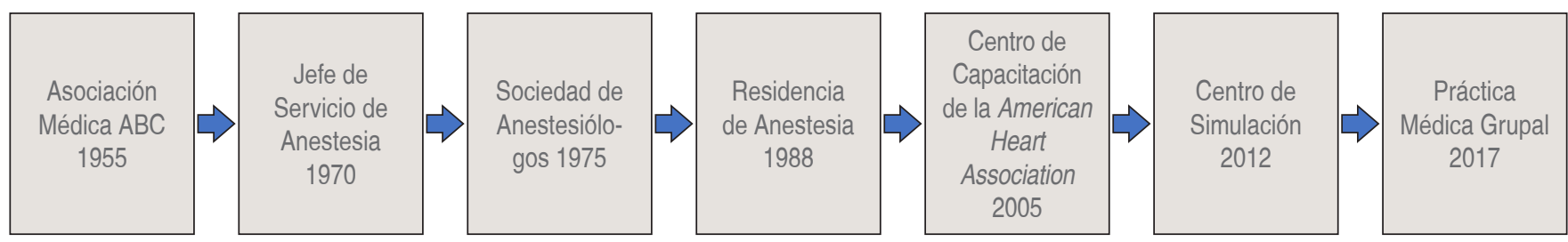

Figura 2: Línea de tiempo de eventos organizados por anestesiólogos dentro del Centro Médico ABC.

en todo momento y teniendo cada vez más participación en el periodo perioperatorio. Ocupamos muchos espacios, ya que estamos presentes en hemodinamia, urgencias, terapia intensiva, quirófano con todas las especialidades, ginecoobstetricia, pediatría, rayos $\mathrm{X}$, endoscopia, radioterapia, beneficencia, dolor, cuidados paliativos, urología, robótica y hasta medio ambiente y salud mental.

El Centro Médico ABC está en su tercer siglo (si tomamos en cuenta que se inicia a finales de 1800 , durante todo 1900 y ya con 20 años del actual siglo), existen muchos retos dentro de nuestra especialidad: transformar la formación académica a través de los simuladores y simulación, con apoyos cognitivos visuales, auditivos y sensoriales, monitores no invasivos que aporten mayor información del interior del cuerpo, sin olvidar el impacto ambiental y realizar una práctica consciente de los residuos tóxicos para el ambiente, especialmente los de un solo uso, garantizando en todo momento la seguridad del paciente.

Por último, cuando comprendamos que la anestesia no existiría sin nuestra relación con los otros seres que habitan el planeta, ya que la belladona sale del árbol floripondio, los relajantes musculares de las plantas curare, el opio de la amapola, el propofol del huevo de gallina, la insulina de los cerdos, etcétera, probablemente entonces más anestesiólogos se involucren también en todos los espacios de la institución para ser conscientes de la necesidad de transformar la práctica de la medicina sin dañar al planeta.

En conclusión, todo mi agradecimiento a maestros y compañeros, mujeres y hombres, que aportando cada uno su talento especial, han hecho que nuestra especialidad dentro de esta institución sea valorada y respetada. Que a pesar de nuestras diferencias hemos encontrado un camino cordial y amable para encontrarnos en un objetivo común: practicar nuestra especialidad actualizados con los mejores recursos, fomentando la educación de todo el equipo quirúrgico para que el paciente esté seguro y bien atendido mientras está en nuestra sala de operaciones.

\section{AGRADECIMIENTOS}

Los doctores que hicieron posible este artículo con su tiempo para recordar fechas, anécdotas y datos precisos: Pastor Luna, Carlos Hurtado, Hilario Genovés, Marco Antonio Chávez, Jaime Ortega, Francisco Revilla, Leopoldo Torres, Miguel Ángel González V, Rubén Valdespín, Elías Horta, Antonio Covarrubias, Delwyn Cordero, Jesús Cruz, Juan Pablo Sánchez, Rodrigo Rubio, Abraham Gutiérrez, Alejandro Díaz, Ulises Sánchez, Gerardo Bermúdez.

Las doctoras que colaboraron para dar su versión de la misma historia: Estela Melman, Margarita Araujo, Patricia Ochoa, Teresa Esquinca, Socorro Espíritu, Verónica Colin, Ulrike Holler, Vanesa Rodríguez, Mercedes Cendón, Taryn García, Elisa Rionda, Mariana García, Claudia Tomás, Brenda Bernal.

Merecen una mención especial: el enfermero Marcos Hernández que ha colaborado con varios anestesiólogos a lo largo de estos 50 años. Esta historia fue armada en gran parte gracias a su memoria y trabajo continuo dentro de la institución. Las jefas de enfermería: Margarita Trejo (Santa Fe), Margarita Ramírez (Observatorio) y Dolores Carrasco (Dirección) también contribuyeron con fechas y anécdotas.

\section{REFERENCIAS}

1. Seguí VP. Historia de las mujeres en el Centro Médico ABC. An Med Asoc Med Hosp ABC. 2020; 65 (1): 81-85. doi: 10.35366/92924.

2. Bosch MG. Centro Médico ABC. Filantropía y Medicina en México. México: Trilce; 2020. p. 201.

3. Shein M. 50 Aniversario de la Revista Anales Médicos. México: Lito-Grapo; 2006. pp. 40-41. 